Article

\title{
Effects of sentiment and emotion of campaign pitch on crowdfunding performance: A cross-cultural comparison
}

\author{
Xiang Gao ${ }^{*}$, Weige Huang ${ }^{2}$, and Sunghan $\mathrm{Ryu}^{3}$ \\ 1 Research Center of Finance, Shanghai Business School, Shanghai, China; gaoxiang@sbs.edu.cn \\ 2 Wenlan School of Business, Zhongnan University of Economics and Law, Wuhan, China; \\ weige_huang@zuel.edu.cn \\ 3 USC-SJTU Institute of Cultural and Creative Industry, Shanghai Jiao Tong University, Shanghai, China; \\ shryu@sjtu.edu.cn \\ * Correspondence: gaoxiang@sbs.edu.cn.
}

Received: 29 July 2021; Accepted: 8 September 2021; Published: 10 September 2021

\begin{abstract}
This study examines the effects of two extreme sentiments (positive vs. negative) and eight types of emotion (anger, fear, anticipation, trust, surprise, sadness, joy, and disgust) implied by the wording of the campaign pitch on the outcomes of crowdfunding, and how these effects would vary according to cultural differences (Western vs. Eastern). We analyze a sample drawn from 17,289 crowdfunding projects on Kickstarter in the "design" and "technology" category to examine how feelings can affect the success, the number of backers, and pledged amounts for crowdfunding projects. We find three regularities by using propensity score match to map Western campaigns to Eastern ones. First, negative sentiment is adversely associated with the success probability but positively related to backer count in the Eastern culture subsample. Second, specific emotions have a larger impact on crowdfunding success among Western culture projects. Third, the statistical significance and direction of the concerning effects differ across the two cultures. Our findings stay robust when we add more controls in a stepwise way. Hence, the study contributes to the literature and provides suggestions for practitioners by exploring the role of derived feelings in the crowdfunding campaign pitch and the entrepreneurial pitch at the early financing stage.
\end{abstract}

Keywords: Crowdfunding; sentiment; emotion; Kickstarter; campaign pitch; cultural differences

JEL codes: C55; D91; G29; L26

\section{Introduction}

Crowdfunding is a new fundraising practice for creative projects, social causes, and ventures (Mollick, 2014; Ryu, 2019). The funds come from a large number of contributors (a.k.a. backers or supporters of the projects), and each participant contributes a relatively small amount through an online platform. In the past, regular people, compared to a small circle of professionals, found it challenging to gain opportunities to participate in early-stage financing for promising ventures, even when they had the financial resources. Those barriers have been lowered, thanks to the emergence of crowdfunding (Ryu et al., 2020; Shneor \& Munim, 2019; Zheng et al., 2018).

On the one hand, crowdfunding gives individuals and organizations another way to raise capital outside the experts and turn to the crowd to finance their projects (Dern, 2014; Gerber et al., 2012; Ryu \& Kim, 2018). On the other hand, the expansion of crowdfunding is good news for creators with new ideas across disciplines worldwide. Opening immense opportunities for creators apart from the limited channel for financing gives them a new route to success and sustainable development of their projects and career. Despite the win-win scenario, there was a relatively small market for early adopters at the beginning phase of crowdfunding. However, the overall crowdfunding industry has 
been expanding to account for comparable market size with the traditional players such as the venture capital industry. For example, as of March 2020, Kickstarter, a representative crowdfunding platform based in the U.S., has raised $\$ 4.8$ billion from more than 17 million people for 180,000 campaigns created on the basis of both the Western and Eastern culture since its launch in 2009.

Given the importance of crowdfunding, an emerging body of research has focused on the signals delivered by campaign pitches' verbal and non-verbal content (Davis et al., 2017; Kaminski \& Hopp, 2019; Parhankangas \& Renko, 2017), which constitute the basis of our project. But we still do not know how backers feel about campaign pitch content would affect crowdfunding outcomes. The missing element is hence exploring the impacts of campaign pitches' sentimental characteristics.

In this regard, for examining the derivative effects of verbal components, our research project first aims to explore how different sentimental components in a campaign pitch affect crowdfunding success. This first research question also complements the literature examining the role of discourse by creators in the crowdfunding context (Anglin et al., 2018; Parhankangas \& Renko, 2017). However, several essential issues remain unanswered. In specific, it is not clear which sentiment or emotions constitute the crowdfunding discourse. This stream literature rarely addressed the characteristics and components of the inputs. For example, we have little idea whether potential contributors are more attracted to pledge by particular sentiments of campaign pitches. This neglect on the input side is a critical missing in understanding crowdfunding because the content of campaign pitches plays an essential role in reducing risks or uncertainty due to information asymmetry associated with the crowdfunding campaigns. Undoubtedly, more knowledge and awareness of such emotional elements can provide a deeper understanding of what makes successful crowdfunding campaigns.

Moreover, we propose that the magnitude and significance of such impact are associated with the campaign's birthplace or location since emotions are largely determined by differences between Western and Eastern countries and regions in the cultural and socioeconomic environment surrounding creators and backers. This second research question originates from the literature that seeks to explain helping behavior (e.g., contribution to a crowdfunding campaign) due to an interaction between dispositional and situational components (Mendoza et al., 2001). Thus, mechanisms underlying the action of help may vary across cultures since culture can be considered a placeholder for contextual differences. It may be the case that the effects of particular sentiments are more or less likely to be influential in one cultural context than in others. While this literature demonstrates the importance of cultural contexts and the existence of pronounced differences in characteristics and levels of helping behavior between the two cultural groups---collectivists and individualists (Cho \& Kim, 2017), it is an unanswered question how different sentiments of crowdfunding campaign pitches work differently across the two cultural groups. Thus, our research project examines the cross-cultural effects on the relationship between sentiments expressed in a crowdfunding campaign pitch and the corresponding crowdfunding outcome.

For the research objectives, we collected 17,289 crowdfunding projects in the "design" and "technology" category on Kickstarter, one of the leading crowdfunding platforms worldwide. We then drew a sample by matching Western and Eastern culture projects and performed analysis. Specifically, we examine the effects of two extreme sentiments, positive vs. negative sentiments, and eight emotion types, including anger, fear, anticipation, trust, surprise, sadness, joy, and disgust, implied in the campaign pitch on the outcomes of crowdfunding. Besides, we also explore how such effects vary depending on Western-based and Eastern-based campaigns. Using the Propensity Score Matching (PSM) approach and regressive modeling approach, we found some implicative tendencies. First, in the Eastern culture subsample, negative sentiment is adversely associated with crowdfunding success but positively with the backer count. Second, most of the emotions are more influential in the Western subsample than in the Eastern ones regarding campaign success prediction. Finally, the direction, the statistical significance, and the extent of the effects of emotions on the outcomes of crowdfunding differ across the two cultures. While the final status of both Western and Eastern culture crowdfunding projects are affected by emotions delivered by campaign pitch, those feelings seem to only matter in the Eastern culture subsample in terms of the number of backers and the pledged amount conditional on campaign success. Our paper contributes to the crowdfunding literature that explores the roles of 
sentiments in crowdfunding campaign pitches by crowdfunding creators and the venture capital literature on the entrepreneurial pitch at the early stage financing in general. We also add to studies investigating how entrepreneurs can communicate their ventures effectively to stakeholders for providing resources. Practically, creators and crowdfunding platform operators could benefit from the findings in preparing and guiding the better design of crowdfunding campaigns. Most importantly, policymakers can better leverage crowdfunding to promote creativity and innovation in the digital era.

The remainder of the paper is organized as follows. Section 2 presents the data and quantifies the feeling reflected in crowdfunding project descriptions. Section 3 specifies our empirical model. Section 4 presents and discusses empirical results, followed by Section 5 that concludes.

\section{Data Sample and Sentiment Measurement}

For this study, we start with compiling a pool of Kickstarter campaigns from January 2016 through December 2019. Then, we randomly select 25,000 projects from this data pool by restricting campaigns to only those falling into the "design" and "technology" categories. We believe these two categories are most closely related to our research question of investigating the influences of cultural differences. By doing this, undesirable disturbances generated by the biases inherent in the entire pool and by categories such as "arts" and "charity" that are by themselves emotional will be in large part mitigated. Next, we only keep campaigns located in the U.S., Hong Kong, Japan, and Singapore, as campaigns of our chosen categories from other places are relatively sparse. This step reduces our sample size to 17,289 crowdfunding projects, including 12,167 "technology"-category campaigns and 5,122 under the "design" category. For cultural classification, the Eastern culture group includes Hong Kong, Japan, and Singapore, whereas the Western culture group is represented only by campaigns initiated from the U.S. In terms of cultural differences, it is noteworthy that our sample is extremely unbalanced across the two groups or subsamples. We have 16,915 campaigns in the Western culture subsample but only 374 in the Eastern culture subsample. Therefore, we need to match the data to have a balanced sample using the PSM approach described in detail in the next section.

A single observation in our dataset contains the following list of variables divided into the dependent, explanatory, and control variables. The dependent variables include: whether the campaign under concern is successfully funded or not at the end, the number of backers (in thousands of people), and the pledged amount (in millions of U.S. dollars). The explanatory variables are proxies of sentiment and emotion derived from campaign pitch or description (a.k.a. the blurb). Besides utilizing the creator's origin country as the sample dividing criterion, we construct and incorporate several control variables into our regressions. They include the number of words contained in the crowdfunding campaign pitch, the goal amount (in millions of U.S. dollars), campaign creation date, campaign launch date, and the date when the campaign has changed status between various scenarios such as success, failure, pause or cancel, etc.

Next, we quantify the sentiment and emotions expressed by crowdfunding campaign creators. Our study pulls out and presents sentimental and emotional indices by applying a standard R package for sentiment analysis to the wording of crowdfunding campaign pitches (Mohammad \& Turney, 2010). This package utilizes the National Research Council Canada (NRC) Sentiment and Emotion Lexicon, which relates a collection of words with associated ratings on both positive and negative sentiment and associated ratings on a variety of specific emotions. The NRC Sentiment and Emotion Lexicons are separate lexicons, incorporating the widely used Word-Emotion Association Lexicon. Those lexicons have been developed with various applications such as sentiment analysis, product marketing, consumer behavior analysis, and even political campaign analysis. Each lexicon has a list of words and their associations with specific categories of interest, such as emotions (joy, sadness, fear, etc.), sentiment (positive and negative), or color (red, blue, black, etc.). All of the lexicons include entries for English words and can be used to analyze English texts like the crowdfunding campaign pitch in our paper.

Table 1 below reports the summary statistics for the main variables. We can see that the means for final status and pledged amounts are quite different between the two cultural groups. However, for the number of supporters and the number of words, the mean values for the two groups are close. 
For the goal variable, there exists a huge difference between the means of the two groups. And for emotional factors, the means are similar. All variables have intermediate pair-wise correlation and fairly low standard deviations (S.D.).

Table 1. Summary Statistics before the Matching the Western and Eastern Culture Subsamples

\begin{tabular}{ccccccccccc}
\hline & \multicolumn{4}{c}{ Western Culture Subsample } & \multicolumn{5}{c}{ Eastern Cultural Subsample } \\
\hline & Min & Mean & Median & Max & S.D. & Min & Mean & Median & Max & S.D. \\
\hline Final Status & 0 & 0.42 & 0 & 1 & 0.49 & 0 & 0.64 & 1 & 1 & 0.48 \\
No. of Backers & 0 & 0.32 & 0.03 & 78.47 & 1.69 & 0 & 0.33 & 0.1 & 10.12 & 0.85 \\
Pledged Amt. & 0 & 0.04 & 0 & 20.34 & 0.31 & 0 & 0.77 & 0.09 & 146.97 & 7.67 \\
No. of Words & 1 & 18.71 & 20 & 50 & 4.84 & 1 & 17.23 & 18 & 28 & 4.65 \\
Goal & 0 & 0.08 & 0.01 & 100 & 1.3 & 0 & 0.36 & 0.05 & 25 & 1.77 \\
Negative Sent. & 0 & 0.35 & 0 & 5 & 0.63 & 0 & 0.39 & 0 & 4 & 0.76 \\
Positive Sent. & 0 & 1.4 & 1 & 8 & 1.19 & 0 & 1.43 & 1 & 6 & 1.18 \\
Anger & 0 & 0.18 & 0 & 3 & 0.43 & 0 & 0.24 & 0 & 4 & 0.52 \\
Anticipation & 0 & 0.58 & 0 & 5 & 0.76 & 0 & 0.63 & 0 & 4 & 0.77 \\
Disgust & 0 & 0.08 & 0 & 3 & 0.29 & 0 & 0.07 & 0 & 1 & 0.26 \\
Fear & 0 & 0.21 & 0 & 6 & 0.49 & 0 & 0.28 & 0 & 4 & 0.61 \\
Joy & 0 & 0.52 & 0 & 6 & 0.75 & 0 & 0.52 & 0 & 4 & 0.76 \\
Sadness & 0 & 0.19 & 0 & 4 & 0.44 & 0 & 0.17 & 0 & 2 & 0.39 \\
Surprise & 0 & 0.23 & 0 & 4 & 0.49 & 0 & 0.29 & 0 & 3 & 0.56 \\
Trust & 0 & 0.68 & 0 & 5 & 0.83 & 0 & 0.75 & 1 & 5 & 0.85 \\
\hline
\end{tabular}

Note. There are 16,915 observations in the unmatched Western subsample; and only 374 observations in the Eastern culture subsample.

In terms of a preliminary investigation of our sample, we find that crowdfunding campaign pitches mostly have positive tones, as expected. Moreover, pitches that deliver emotions like anticipation, joy, and trust are associated with higher funding values. Utilizing the R-package "syuzhet" function, we conduct a simple polarity analysis and extract ratings for two extreme sentiments (namely, negative vs. positive) and eight basic emotions (namely, anger, fear, anticipation, trust, surprise, sadness, joy, and disgust) based on the content of each campaign pitch. Given these ratings, even before going through formal econometric models, we can spot associations between our ratings and campaign outcome variables using crude graph tools and a correlation matrix. Further, we are also able to examine how the associations vary according to cultural differences, as the Kickstarter platform provides services in all regions around the world. Particularly, we will compare campaigns created in the U.S. (representing the Western culture) and their counterparties created in Hong Kong, Japan, and Singapore (representing the Eastern culture).

\section{Regression Model Specification}

We do encounter a challenging task when running regressions in two cultural groups separately. It is challenging because the Western region group outnumbers the Eastern region group concerning the number of observations. Actually, in our sample, there are only 2.2 percentages of campaigns belonging to the Eastern group. In other words, crowdfunding campaigns' locations are extremely unbalanced between the Western vs. Eastern subsamples. To compare the estimation results of the two groups consistently, we resort to the PSM approach and then pair up one Western campaign with a corresponding Eastern one according to emotional factors and campaign characteristics. The Rpackage "matchit" is employed to implement the match. In specific, this function estimates the propensity score for each campaign project and then performs the best match among two designated groups, which are the Western vs. Eastern cultural group, according to some criteria of choice, which in our case is the "nearest" rule.

The PSM method aims to solve the unbalanced sample problem by finding pairs of observations that have very similar propensity scores but that differ in their cultural backgrounds. The disadvantage of such treatment is that we have a much smaller number of observations. However, this disadvantage does not compromise our results as the final sample results from random selection; the PSM method 
alleviates the potential endogeneity problem. After the matching process, we obtain 748 observations in total (374 for each group). By doing this, the estimated coefficients for the two cultural groups can now be directly compared on the same basis as the matched Western- and Eastern-culture subsamples are similar to each other in every way except for their crowdfunding results.

The logit model is adopted when the dependent variable is the final status of a campaign. And the ordinary least squares model is utilized when the dependent variable is either the number of campaign supporters or the pledged amounts raised during the campaign course. The regressive empirical methodology takes the following specification:

$$
\text { Outcome }_{i}=\alpha+\boldsymbol{\beta}^{\prime} \boldsymbol{X}_{i}+\boldsymbol{\gamma}^{\prime} \boldsymbol{Z}_{i}+\varepsilon_{i},
$$

where Outcome $_{i}$ is one of the three aspects of crowdfunding project i's outcome: whether the final status is success or failure, the number of backers, and the amounts of funds raised. $\boldsymbol{X}_{i}$ is either a bivariate vector of sentiment or a vector of emotions. $\operatorname{In} \boldsymbol{Z}_{i}$, we include an array of campaign-specific controls such as the number of words used to describe the crowdfunding campaign projects, the goal amount, the creation and launch date, and a change in status during the fundraising course. $\varepsilon_{i}$ is the usual error term. We expect to estimate a pair of $\boldsymbol{\beta}$ vector coefficients for the two cultural subsamples and make a comparison of their signs and magnitude across these two subsamples. Hopefully, we can discover some patterns of how cultural positions determine the effect of sentiment and emotions on crowdfunding projects carried out on the Kickstarter platform.

\section{Results}

The regression results with alternative dependent variables are summarized and interpreted below in a consecutive of three subsections. After presenting the main findings in each subsection, we continue to discuss their relationship with those documented in the extant literature and comment on their economic meaning and practical implication from the perspective of cultural difference.

\subsection{Final Status}

Using the whole sample after matching Western and Eastern campaigns, we first regress the final status on sentiment proxies. Table 2 presents the estimation results of the impacts imposed by positive and negative pitch sentiment on whether the underlying campaign will succeed for the Western and Eastern subsample, respectively. As can be seen, in all specifications, neither positive nor negative sentiment seems to be correlated with campaigns created in the Western world. In contrast, Eastern campaigns are more likely to fail if their pitch discloses some sort of negative sentiment. However, such an effect is not found on the positive side, i.e., delivering a positive sentiment does not help Eastern creators to get fully financed.

Then, we replace the two extreme sentiment statistics with eight indicators of different aspects of emotions and re-run the above exercises. Table 3 includes the corresponding results for the group of Western vs. Eastern sample campaigns. For both cultural environments, expressing anger when writing a campaign pitch has no significant impact on final status. In the Western group, all other emotions except for anger exhibit statistically significant associations with success probability. While emotions like anticipation, fear, surprise, and trust are negative predictors of the final status, stronger emotions of disgust, joy, and sadness turn out to forecast success positively.

These Western patterns change significantly in the Eastern group. Moreover, their signs and significance stay unchanged when we add more controls in a stepwise way. Campaigns cultivated out of the Eastern setup are less emotionally affected. The tendency is evident by more insignificant estimated coefficients, e.g., anticipation, joy, and trust, in addition to anger. For another, like in the Western group, the fear and disgust emotion is, respectively, negative and positive significant at the $5 \%$ level; unlike Western campaigns, the surprise factor becomes positively correlated with success at the $1 \%$ level, and the sadness coefficient is now negative but only at a $10 \%$ significance level. 
Table 2. Impacts of Bipolar Sentiment on Final Status (Western vs. Eastern Culture)

\begin{tabular}{|c|c|c|c|c|c|}
\hline & (1) & (2) & (3) & (4) & (5) \\
\hline \multicolumn{6}{|c|}{ Panel A: Western Culture Subsample } \\
\hline Negative Sentiment & -0.19 & -0.14 & -0.15 & -0.17 & -0.14 \\
\hline Positive Sentiment & -0.08 & -0.06 & -0.07 & -0.02 & -0.04 \\
\hline Number of Words & & $-0.06^{* *}$ & $-0.06^{* *}$ & -0.03 & -0.03 \\
\hline Goal & & & -1.50 & -0.47 & -0.72 \\
\hline Intercept & -0.11 & $0.93^{*}$ & $0.98^{*}$ & -14.29 & -15.31 \\
\hline Creation Date & No & No & No & Yes & Yes \\
\hline Launch Date & No & No & No & Yes & Yes \\
\hline \multirow[t]{2}{*}{ Changed Status } & No & No & No & No & Yes \\
\hline & (6) & (7) & (8) & (9) & (10) \\
\hline \multicolumn{6}{|c|}{ Panel B: Eastern Culture Subsample } \\
\hline Negative Sentiment & $-0.25^{*}$ & $-0.24^{*}$ & $-0.24^{*}$ & $-0.32^{* *}$ & $-0.29^{*}$ \\
\hline Positive Sentiment & 0.06 & 0.06 & 0.06 & 0.03 & 0.03 \\
\hline Number of Words & & -0.01 & -0.02 & -0.01 & -0.01 \\
\hline Goal & & & $-0.17^{*}$ & -0.21 & -0.21 \\
\hline Intercept & $0: 60^{* * *}$ & $0.78^{*}$ & $1.04^{* *}$ & 0.22 & -0.09 \\
\hline Creation Date & No & No & No & Yes & Yes \\
\hline Launch Date & No & No & No & Yes & Yes \\
\hline Changed Status & No & No & No & No & Yes \\
\hline
\end{tabular}

Note. There are 374 observations in the sample. ${ }^{*} \mathrm{p}<0.1 ;{ }^{* *} \mathrm{p}<0.05 ;{ }^{* * *} \mathrm{p}<0.01$.

Overall, the success of Western campaigns depends more on specific emotions rather than simple polarized sentiment. In comparison, when pitching crowdfunding campaigns in the Eastern region, creators should avoid picturing a negative sentiment and be careful with emotions like surprise and fear. One point that merits a note is that certain emotions, e.g., disgust, have counter-intuitive relation with campaign success. The reason might be what matters here most is the strength of emotions, probably not the actual taste of feeling. Stronger feelings attract more views on the crowdfunding platform, leading to a larger conversion ratio of turning visitors into participants.

\subsection{Number of Backers or Supporters}

To further understand the implication of sentiment and emotions, we estimate their effect on the number of backers who have supported crowdfunding campaigns, conditional on the final status being a success. For this purpose, we keep only successful campaigns. The sample size of both the Western and Eastern campaigns reduces from an equal number of 374 to 150 and 240, respectively. Table 4 shows the impacts of sentiment on the number of backers for the two culture groups.

The bipolar sentiment seems to be uncorrelated with the number of supporters for crowdfunding projects initiated in the Western world. Turning to the Eastern culture subsample, negative sentiment consistently matters for the number of backers for campaigns created in the Eastern culture. However, the corresponding coefficient estimates in front of negative sentiment are statistically significant only at the $10 \%$ level. As for coefficient estimates before positive sentiment in the Eastern group, their 5\% significance disappears after controlling for the creation and launching date variables.

From the point of view of economic meanings, this result about the number of supporters has important implications when the concerning crowdfunding projects aim at evaluating the market potential and drawing the attention of customers. In specific, extreme sentiment expressed in Eastern culture projects, irrespective of positive and negative, can help expand the reach of crowdfunding campaigns and the associated products or services to the targeted customer base. 
Table 3. Impacts of a List of Different Emotions on Final Status (Western vs. Eastern Culture)

\begin{tabular}{|c|c|c|c|c|c|}
\hline & (1) & $(2)$ & (3) & (4) & (5) \\
\hline \multicolumn{6}{|c|}{ Panel A: Western Culture Subsample } \\
\hline Anger & 0.12 & 0.16 & 0.20 & 0.04 & -0.14 \\
\hline Anticipation & $-0.37^{*}$ & $-0.34^{*}$ & $-0.34^{*}$ & $-0.44^{* *}$ & $-0.43^{* *}$ \\
\hline Disgust & $0.92^{* * *}$ & $1.00^{* * *}$ & $0.99^{* * *}$ & $1.24^{* * *}$ & $1.27^{* * *}$ \\
\hline Fear & $-2.85^{* * *}$ & $-2.88^{* * *}$ & $-2.90^{* * *}$ & $-3.04^{* * *}$ & $-2.79^{* * *}$ \\
\hline Joy & $0.61^{* * *}$ & $0.65^{* * *}$ & $0.65^{* * *}$ & $0.83^{* * *}$ & $0.82^{* * *}$ \\
\hline Sadness & $1.85^{* * *}$ & $1.89^{* * *}$ & $1.88^{* * *}$ & $2.08^{* * *}$ & $2.00^{* * *}$ \\
\hline Surprise & $-1.41^{* * *}$ & $-1.54^{* * *}$ & $-1.56^{* * *}$ & $-1.83^{* * *}$ & $-1.77^{* * *}$ \\
\hline Trust & $-0.90^{* * *}$ & $-0.89^{* * *}$ & $-0.90^{* * *}$ & $-1.04^{* * *}$ & $-1.02^{* * *}$ \\
\hline Number of Words & & $0.06^{* *}$ & $-0.06^{* *}$ & -0.04 & -0.04 \\
\hline Goal & & & 1.85 & -0.78 & -0.91 \\
\hline Intercept & $-2.73^{* * *}$ & $-1.75^{* *}$ & $-1.68^{* *}$ & -16.83 & -17.79 \\
\hline Creation Date & No & No & No & Yes & Yes \\
\hline Launch Date & No & No & No & Yes & Yes \\
\hline \multirow[t]{2}{*}{ Changed Status } & No & No & No & No & Yes \\
\hline & (6) & (7) & $(8)$ & (9) & (10) \\
\hline \multicolumn{6}{|c|}{ Panel B: Eastern Culture Subsample } \\
\hline Anger & 0.14 & 0.14 & 0.11 & -0.07 & -0.02 \\
\hline Anticipation & $0.39 *$ & $0.39 *$ & $0.41^{* *}$ & 0.32 & 0.34 \\
\hline Disgust & $1.35^{* *}$ & $1.32^{* *}$ & $1.39^{* *}$ & $1.37^{* *}$ & $1.30^{* *}$ \\
\hline Fear & $-0.54^{* *}$ & $-0.54^{* *}$ & $-0.56^{* *}$ & $-0.54^{* *}$ & $-0.51^{* *}$ \\
\hline Joy & -0.09 & -0.08 & -0.09 & -0.11 & -0.08 \\
\hline Sadness & $-0.58^{*}$ & $-0.56^{*}$ & $-0.56^{*}$ & $-0.67^{*}$ & $-0.71^{*}$ \\
\hline Surprise & $0.50^{* *}$ & $0.52^{* *}$ & $0.56^{* *}$ & $0.74^{* * *}$ & $0.78^{* * *}$ \\
\hline Trust & 0.13 & 0.14 & 0.14 & 0.18 & 0.16 \\
\hline Number of Words & & 0.02 & -0.03 & -0.03 & -0.03 \\
\hline Goal & & & $0.21^{* *}$ & $-0.26^{* *}$ & $-0.28^{* *}$ \\
\hline Intercept & $0.33^{* *}$ & 0.66 & $0.95^{* *}$ & 0.15 & -0.19 \\
\hline Creation Date & No & No & No & Yes & Yes \\
\hline Launch Date & No & No & No & Yes & Yes \\
\hline Changed Status & No & No & No & No & Yes \\
\hline
\end{tabular}

Note. There are 374 observations in the sample. ${ }^{*} \mathrm{p}<0.1 ;{ }^{* *} \mathrm{p}<0.05 ;{ }^{* * *} \mathrm{p}<0.01$.

Table 4. Impacts of Bipolar Sentiment on Number of Backers (Western vs. Eastern Culture)

\begin{tabular}{|c|c|c|c|c|c|}
\hline & (1) & $(2)$ & (3) & (4) & (5) \\
\hline \multicolumn{6}{|c|}{ Panel A: Western Culture Subsample } \\
\hline Negative Sentiment & -0.72 & -0.78 & -0.23 & -0.25 & -0.32 \\
\hline Positive Sentiment & -0.24 & -0.34 & 0.13 & -0.24 & -0.21 \\
\hline Number of Words & & 0.11 & -0.01 & 0.05 & 0.04 \\
\hline Goal & & & $62.74^{* * *}$ & $62.76^{* * *}$ & $63.47^{* * *}$ \\
\hline Intercept & 2.29 & 0.44 & -0.41 & -0.43 & -0.42 \\
\hline Creation Date & No & No & No & Yes & Yes \\
\hline Launch Date & No & No & No & Yes & Yes \\
\hline \multirow[t]{2}{*}{ Changed Status } & No & No & No & No & Yes \\
\hline & (6) & (7) & (8) & (9) & (10) \\
\hline \multicolumn{6}{|c|}{ Panel B: Eastern Culture Subsample } \\
\hline Negative Sentiment & $0.18^{*}$ & $0.16^{*}$ & $0.17^{*}$ & $0.18^{*}$ & $0.19^{*}$ \\
\hline Positive Sentiment & $0.12^{* *}$ & $0.11^{* *}$ & $0.11^{* *}$ & 0.09 & 0.08 \\
\hline Number of Words & & 0.02 & $0.03^{*}$ & 0.02 & $0.03^{*}$ \\
\hline Goal & & & 0.09 & 0.09 & 0.10 \\
\hline Intercept & $0.24^{* *}$ & -0.11 & -0.22 & -0.18 & -0.32 \\
\hline Creation Date & No & No & No & Yes & Yes \\
\hline Launch Date & No & No & No & Yes & Yes \\
\hline Changed Status & No & No & No & No & Yes \\
\hline
\end{tabular}

Note. There are 150 and 240 obs. in columns (1)-(5) and (6)-(10), respectively. ${ }^{*} \mathrm{p}<0.1 ;{ }^{* *} \mathrm{p}<0.05 ;{ }^{* * *} \mathrm{p}<0.01$. 
In terms of the explanatory power of eight basic emotions, the results are presented in Table 5. In the Western region group, all eight types of emotions are uncorrelated with the size of the backer crowd. In contrast, the reactions of backers for projects cultivated in Eastern culture countries are of economically large magnitude. The number of people supporting Eastern crowdfunding campaigns increases significantly with two emotions, more with the disgust factor and less with the surprise factor. Specifically, the estimated coefficient of the disgust factor is roughly three times larger than that of the surprise factor. We suspect that such an effect of the disgust factor relates to curiosity, and the core of expressing feelings in crowdfunding projects lies in the degree of strength of emotions, not in the type or meaning of emotions.

Table 5. Impacts of a List of Different Emotions on Number of Backers (Western vs. Eastern Culture)

\begin{tabular}{|c|c|c|c|c|c|}
\hline & (1) & (2) & (3) & (4) & (5) \\
\hline \multicolumn{6}{|c|}{ Panel A: Western Culture Subsample } \\
\hline Anger & 1.92 & 1.98 & -1.05 & -2.10 & -1.92 \\
\hline Anticipation & -0.05 & -0.09 & 0.47 & 0.39 & 0.45 \\
\hline Disgust & -0.90 & -0.96 & -0.25 & -0.09 & -0.22 \\
\hline Fear & 1.16 & 1.23 & 1.28 & 0.96 & 1.11 \\
\hline Joy & 0.78 & 0.68 & 0.53 & 0.19 & 0.25 \\
\hline Sadness & -1.08 & -1.12 & -0.29 & 0.19 & 0.04 \\
\hline Surprise & -0.67 & -0.52 & -0.11 & 0.06 & -0.02 \\
\hline Trust & -1.57 & -1.57 & -0.71 & -0.02 & -0.06 \\
\hline Number of Words & & 0.09 & -0.02 & 0.02 & 0.01 \\
\hline Goal & & & $62.93^{* * *}$ & $64.05^{* * *}$ & $64.77^{* * *}$ \\
\hline Intercept & 2.40 & 0.91 & -0.33 & -1.24 & -0.97 \\
\hline Creation Date & No & No & No & Yes & Yes \\
\hline Launch Date & No & No & No & Yes & Yes \\
\hline \multirow[t]{2}{*}{ Changed Status } & No & No & No & No & Yes \\
\hline & (6) & (7) & (8) & (9) & (10) \\
\hline \multicolumn{6}{|c|}{ Panel B: Eastern Culture Subsample } \\
\hline Anger & -0.11 & -0.11 & -0.10 & -0.03 & -0.002 \\
\hline Anticipation & -0.06 & -0.06 & -0.06 & -0.07 & -0.04 \\
\hline Disgust & $0.73^{* * *}$ & $0.72^{* * *}$ & $0.72^{* * *}$ & $0.75^{* * *}$ & $0.71^{* * *}$ \\
\hline Fear & -0.12 & -0.11 & -0.11 & -0.11 & -0.09 \\
\hline Joy & 0.03 & 0.03 & 0.03 & 0.01 & 0.04 \\
\hline Sadness & -0.09 & -0.08 & -0.09 & -0.12 & -0.09 \\
\hline Surprise & $0.26^{* *}$ & $0.24^{* *}$ & $0.23^{*}$ & $0.23^{*}$ & $0.25^{* *}$ \\
\hline Trust & 0.11 & 0.10 & 0.11 & 0.09 & 0.04 \\
\hline Number of Words & & 0.02 & 0.02 & 0.02 & 0.02 \\
\hline Goal & & & 0.07 & 0.08 & 0.08 \\
\hline Intercept & $0.32^{* * *}$ & 0.03 & -0.05 & -0.16 & -0.38 \\
\hline Creation Date & No & No & No & Yes & Yes \\
\hline Launch Date & No & No & No & Yes & Yes \\
\hline Changed Status & No & No & No & No & Yes \\
\hline
\end{tabular}

Note. There are 150 and 240 observations in the samples used for columns (1)-(5) and (6)-(10), respectively. * $\mathrm{p}<0.1 ;{ }^{* *} \mathrm{p}<0.05 ;{ }^{* * *} \mathrm{p}<0.01$.

In sum, the number of supporters serves as a key crowdfunding characteristic as entrepreneurs often employ online platforms such as GoFundMe, Kickstarter, and Indiegogo to test the market with prototype products. More supporters equate to a potentially larger market share, attracting future rounds of venture capital money. Our results add to the extant literature by showing that stronger sentiment or emotions might help attain this goal for projects launched in the Eastern culture. As a result, in the Western culture, online fund-raisers could pay less attention to supporter feelings. 


\subsection{Pledged Amounts}

Like the previous subsection, we only use campaigns that have accomplished their fundraising goals for our analysis as the expectation is that unsuccessful ones are required to return money to supporters. On the Kickstarter platform, creators release their campaign advertisement by setting a goal amount as the last step. The policy is as follows: if this amount to be raised is not achieved, then the campaign under concern is considered unsuccessful, and consequently, all money pledged till maturity will be returned to backers. If the goal amount is fully raised, it is a successful campaign, and the actual amount pledged usually exceeds the goal amount.

Table 6 summarizes the impacts of sentiment on pledged amounts for the Western and Eastern cultural groups, respectively. Table 7 demonstrates how the eight basic emotions extracted from pitch wording affect pledged amounts for campaigns originating from the Western and Eastern regions, respectively.

Table 6. Impacts of Bipolar Sentiment on Pledged Amount (Western vs. Eastern Culture)

\begin{tabular}{|c|c|c|c|c|c|}
\hline & (1) & (2) & (3) & (4) & (5) \\
\hline \multicolumn{6}{|c|}{ Panel A: Western Culture Subsample } \\
\hline Negative Sentiment & -0.13 & -0.14 & -0.04 & -0.02 & -0.03 \\
\hline Positive Sentiment & -0.03 & -0.04 & 0.04 & -0.02 & -0.02 \\
\hline Number of Words & & 0.02 & -0.003 & 0.01 & 0.01 \\
\hline Goal & & & $12.03^{* * *}$ & $12.03^{* * *}$ & $12.13^{* * *}$ \\
\hline Intercept & 0.38 & 0.03 & -0.13 & -0.16 & -0.16 \\
\hline Creation Date & No & No & No & Yes & Yes \\
\hline Launch Date & No & No & No & Yes & Yes \\
\hline \multirow[t]{2}{*}{ Changed Status } & No & No & No & No & Yes \\
\hline & (6) & (7) & (8) & (9) & (10) \\
\hline \multicolumn{6}{|c|}{ Panel B: Eastern Culture Subsample } \\
\hline Negative Sentiment & -0.36 & -0.35 & -0.23 & -0.36 & -0.35 \\
\hline Positive Sentiment & 0.65 & 0.65 & 0.74 & 0.59 & 0.58 \\
\hline Number of Words & & -0.02 & 0.14 & 0.16 & 0.16 \\
\hline Goal & & & $2.92^{* * *}$ & $2.99^{* * *}$ & $3.00^{* * *}$ \\
\hline Intercept & 0.27 & 0.56 & -2.99 & -3.87 & -3.88 \\
\hline Creation Date & No & No & No & Yes & Yes \\
\hline Launch Date & No & No & No & Yes & Yes \\
\hline Changed Status & No & No & No & No & Yes \\
\hline
\end{tabular}

Note. There are 150 and 240 observations in the samples used for columns (1)-(5) and (6)-(10), respectively. * $\mathrm{p}<0.1 ;{ }^{* *} \mathrm{p}<0.05 ;{ }^{* *} \mathrm{p}<0.01$.

As can be seen from these two tables, although the goal amount is positively correlated with the pledged amount, we find no significant linkages between either sentiment or emotion and the pledged amount. Regarding economic interpretations, setting a larger goal amount is endogenously determined by the higher quality of the crowdfunding projects and the higher level of confidence that the fundraiser had in his/her project. The prevailing statistical insignificance of our concerned emotional coefficients illustrates the limit of the capability of embedding emotions in campaign pitches---it does help creators attain their goals, but it does not determine how much funds they will raise eventually. Cultural differences play a much lesser role here.

\section{Discussions and Conclusions}

Crowdfunding is still in its early stages, but it contains potential and capabilities in addressing existing biases in financing different projects. Because of the importance of crowdfunding, researchers from different disciplines have studied the phenomenon of crowdfunding. Crowdfunding literature has focused on essential factors influencing individual contributors' funding decisions and what drives successful crowdfunding campaigns, including the quality of campaign/creator, social influence/social networks, crowdfunding campaign design, and information on campaign progress. Among others, 
one specific stream of crowdfunding research focuses on the signals from the verbal and sentimental components of campaign pitches (Davis et al., 2017; Kaminski \& Hopp, 2019; Parhankangas \& Renko, 2017). More knowledge and awareness of the components can provide a deeper understanding of making successful crowdfunding campaigns.

Adding knowledge to the literature, our paper employs an exploratory approach resting on machine learning methods in mining data of sentimental and emotional components to examine the effects on crowdfunding outcomes. This approach is advantageous to traditional methods because there are growing volumes and varieties of available data for crowdfunding projects. Textual content is the most accessible one, enabling computational processing that is cheaper, more powerful, and affordable data storage. These features mean it is possible to quickly and automatically produce models that can analyze more critical, more complex data and deliver faster, more accurate results even on an enormous scale. Furthermore, by building precise models, a creator has a better chance of pitching the project high with profitable opportunities or avoiding unknown risks. Our research adds to these streams by looking at the relationship between the pitch's sentimental and emotional aspects and three key campaign outcome variables.

Table 7. Impacts of a List of Different Emotions on Pledged Amount (Western vs. Eastern Culture)

\begin{tabular}{|c|c|c|c|c|c|}
\hline & (1) & $(2)$ & (3) & (4) & (5) \\
\hline \multicolumn{6}{|c|}{ Panel A: Western Culture Subsample } \\
\hline Anger & 0.38 & 0.39 & -0.19 & -0.47 & -0.44 \\
\hline Anticipation & 0.02 & 0.01 & 0.11 & 0.09 & 0.10 \\
\hline Disgust & -0.13 & -0.15 & -0.01 & 0.05 & 0.02 \\
\hline Fear & 0.21 & 0.22 & 0.23 & 0.18 & 0.22 \\
\hline Joy & 0.21 & 0.19 & 0.16 & 0.09 & 0.10 \\
\hline Sadness & -0.17 & -0.18 & -0.02 & 0.09 & 0.07 \\
\hline Surprise & -0.20 & -0.17 & -0.09 & -0.03 & -0.05 \\
\hline Trust & -0.42 & -0.42 & -0.26 & -0.09 & -0.09 \\
\hline Number of Words & & 0.02 & -0.004 & 0.003 & 0.003 \\
\hline Goal & & & $12.02^{* * *}$ & $12.26^{* * *}$ & $12.36^{* * *}$ \\
\hline Intercept & 0.34 & 0.06 & -0.18 & -0.38 & -0.33 \\
\hline Creation Date & No & No & No & Yes & Yes \\
\hline Launch Date & No & No & No & Yes & Yes \\
\hline \multirow[t]{2}{*}{ Changed Status } & No & No & No & No & Yes \\
\hline & (6) & $(7)$ & (8) & (9) & (10) \\
\hline \multicolumn{6}{|c|}{ Panel B: Eastern Culture Subsample } \\
\hline Anger & -1.04 & -1.04 & -0.74 & -0.51 & -0.49 \\
\hline Anticipation & 0.17 & 0.17 & 0.28 & 0.28 & 0.34 \\
\hline Disgust & 0.11 & 0.12 & 0.20 & -0.02 & -0.06 \\
\hline Fear & -0.41 & -0.41 & -0.26 & -0.51 & -0.49 \\
\hline Joy & 0.61 & 0.61 & 0.53 & 0.29 & 0.34 \\
\hline Sadness & -0.21 & -0.21 & -0.64 & -0.33 & -0.23 \\
\hline Surprise & 1.29 & 1.30 & 0.95 & 0.99 & 1.00 \\
\hline Trust & -1.05 & -1.05 & -0.98 & -1.17 & -1.25 \\
\hline Number of Words & & -0.01 & 0.15 & 0.17 & 0.17 \\
\hline Goal & & & $2.84^{* * *}$ & $2.93^{* * *}$ & $2.94^{* * *}$ \\
\hline Intercept & 1.40 & 1.58 & -1.85 & -3.34 & -3.39 \\
\hline Creation Date & No & No & No & Yes & Yes \\
\hline Launch Date & No & No & No & Yes & Yes \\
\hline Changed Status & No & No & No & No & Yes \\
\hline
\end{tabular}

Note. There are 150 and 240 observations in the samples used for columns (1)-(5) and (6)-(10), respectively. ${ }^{*}$ $\mathrm{p}<0.1 ;{ }^{* *} \mathrm{p}<0.05 ;{ }^{* *} \mathrm{p}<0.01$.

More broadly, we contribute to the literature on verbal and non-verbal components of entrepreneurial discourse examines how entrepreneurs communicate about their ventures and themselves is essential for persuading stakeholders to provide resources (Roundy \& Asllani, 2018). A stream of studies suggests that content and the appropriate language use should help obtain resources 
by delivering comprehensive messages for an entrepreneur (Lounsbury \& Glynn 2001). Our results complement their findings by pointing out that language matters through the mechanism of emotional perception, and this mechanism differs across different cultural circumstances. Given the above being said, these entrepreneurial discourses shape perceptions about the potential of ventures and thus improve the likelihood of success (Gafni et al., 2019). Our results demonstrate that, in addition to the success or failure outcome, the size of the supporting crowd and the amount of the pledged funds are also associated with such perceptions. Entrepreneurial discourse is not merely delivering the message, it plays a formative role in enabling entrepreneurs to build and scale ventures. All in all, our results corroborate this consensus emphasized by Lounsbury and Glynn (2001) and Roundy and Asllani (2018), and add an element of cultural difference to it.

The findings also generate practical implications for crowdfunding platforms and creators by guiding them to write to win fundraising campaigns with the right sentiment and emotions according to cultural immersion. Moreover, the potential practical implications of this research project allow us to look beyond crowdfunding. The proposed method can be helpful for structuring and sorting available information. For the long term, our research team expects a more advanced system that is competitive to the human capabilities of linguistic and sentimental information processing in crowdsourcing decision making. There should be many opportunities to employ machine learning techniques to predict the potential and, thus, future outcomes for entrepreneurial projects. Therefore, illumining the complex nature of entrepreneurial discourses offers essential academic as well as practical implications.

Author Contributions: Conceptualization, Xiang Gao and Weige Huang; methodology, Weige Huang; software, Weige Huang; validation, Xiang Gao, Weige Huang, and Sunghan Ryu; formal analysis, Sunghan Ryu; investigation, Weige Huang; data curation, Weige Huang; writing-original draft preparation, Xiang Gao; writing - review and editing, Sunghan Ryu; project administration, Xiang Gao. All authors have read and agreed to the published version of the manuscript.

Funding: This research was supported by the Fundamental Research Funds for the Central Universities, Zhongnan University of Economics and Law, grant number 2722021BZ042.

Acknowledgments: We thank the editor and an anonymous reviewer for their helpful comments that improved the paper a lot. All remaining errors are our own.

Conflicts of Interest: The authors declare no conflict of interest.

\section{References}

Anglin, A.H., Short, J.C., Drover, W., Stevenson, R.M., McKenny, A.F., \& Allison, T.H. (2018). The power of positivity? The influence of positive psychological capital language on crowdfunding performance. Journal of Business Venturing, 33(4), 470-492. https://doi.org/10.1016/j.jbusvent.2018.03.003

Cho, M., \& and Kim, G. (2017). A cross-cultural comparative analysis of crowdfunding projects in the United States and South Korea. Computers in Human Behavior, 72, 312-320. https://doi.org/10.1016/j.chb.2017.03.013

Davis, B.C., Hmieleski, K.M., Webb, J.W., \& Coombs, J.E. (2017). Funders' positive affective reactions to entrepreneurs' crowdfunding pitches: The influence of perceived product creativity and entrepreneurial passion. Journal of Business Venturing, 32(1), 90-106. https://doi.org/10.1016/j.jbusvent.2016.10.006

Dern, D.P. (2014). Crowdfunding for Hardware. IEEE Spectrum, 52(1), 22-23. Retrieved August 31, 2021, from spectrum.IEEE.org website: https://spectrum.iee.org/how-to-crowdfund-your-hardware-startup\#togglegdpr

Gafni, H., Marom, D., \& Sade, O. (2019). Are the life and death of an early-stage venture indeed in the power of the tongue? Lessons from online crowdfunding pitches. Strategic Entrepreneurship Journal, 13(1), 3-23. https://doi.org/10.1002/sej.1293

Gerber, E.M, Hui, J.S., \& Kuo, P.-Y. (2012). Crowdfunding: Why people are motivated to post and fund projects on crowdfunding platforms. Proceedings of the International Workshop on Design, Influence, and Social Technologies: Techniques, Impacts and Ethics 2011 (Vol. 2, pp. 10-13). Retrieved August 31, 2021, from researchgate.net website: https://www.researchgate.net/publication/261359489_Crowdfunding_Why_People_are_Motivated_to_Post _and_Fund_Projects_on_Crowdfunding_Platforms 
Kaminski, J.C., \& Hopp, C. (2019). Predicting outcomes in crowdfunding campaigns with textual, visual, and linguistic signals. Small Business Economics, 55, 627-649. https://doi.org/10.1007/s11187-019-00218-w

Lounsbury, M., \& Glynn, M.A. (2001). Cultural entrepreneurship: Stories, legitimacy, and the acquisition of resources. Strategic Management Journal, 22(6-7), 545-564. https://doi.org/10.1002/smj.188

Mendoza-Denton, R., Ayduk, O., Mischel, W., Shoda, Y., \& Testa, A. (2001). Person $\times$ Situation interactionism in self-encoding (Iam...when...): Implications for affect regulation and social information processing. Journal of Personality and Social Psychology, 80(4), 533-544. https://doi.org/10.1037/0022-3514.80.4.533

Mohammad, S.M., \& Turney, P.D. (2010). Emotions evoked by common words and phrases: Using mechanical turk to create an emotion lexicon. Proceedings of the NAACL HLT 2010 Workshop on Computational Approaches to Analysis and Generation of Emotion in Text (pp. 26-34). Retrieved August 31, 2021, from dl.acm.org website: https://dl.acm.org/doi/10.5555/1860631.1860635

Mollick, E. (2014). The dynamics of crowdfunding: An exploratory study. Journal of Business Venturing, 29(1), 1-16. https://doi.org/10.1016/j.jbusvent.2013.06.005

Parhankangas, A., \& Renko, M. (2017). Linguistic style and crowdfunding success among social and commercial entrepreneurs. Journal of Business Venturing, 32(2), 215-236. https://doi.org/10.1016/j.jbusvent.2016.11.001

Roundy, P.T., \& Asllani, A. (2018). Understanding the language of entrepreneurship: An exploratory analysis of entrepreneurial discourse. Journal of Economic and Administrative Sciences, 35(2), 113-127. https://doi.org/10.1108/JEAS-08-2017-0084

Ryu, S. (2019). Beauty of crowdfunding: Blooming creativity and innovation in the digital era. London, England: Routledge.

Ryu, S., \& Kim, Y.-G. (2018). Money is not everything: A typology of crowdfunding project creators. The Journal of Strategic Information Systems, 27(4), 350-368. https://doi.org/10.1016/j.jsis.2018.10.004

Ryu, S., Park, J., Kim, K., \& Kim, Y.-G. (2020). Reward versus altruistic motivations in reward-based crowdfunding. International Journal of Electronic Commerce, 24(2), 159-183. https://doi.org/10.1080/10864415.2020.1715531

S., Rotem, \& Munim, Z. H. (2019). Reward crowdfunding contribution as planned behaviour: An extended framework. Journal of Business Research, 103, 56-70. https://doi.org/10.1016/j.jbusres.2019.06.013

Zheng, H., Xu, B., Zhang, M., \& Wang, T. (2018). Sponsor's cocreation and psychological ownership in rewardbased crowdfunding. Information Systems Journal, 28(6), 1213-1238. https://doi.org/10.1111/isj.12190

(C) 2021 by the authors. This article is an open-access article distributed under the terms and conditions of the Creative Commons Attribution (CC BY) license (http://creativecommons.org/licenses/by/4.0/). 